\title{
Diseño y simulación de un concentrador de flujo para aumentar la potencia eólica disponible en un aerogenerador de baja potencia
}

\section{Design and simulation of a flow concentrator to increase the wind power available in a low power wind turbine}

\author{
Esneider Arciniegas Arenas a \\ Elkin G. Flórez S.b \\ Juan C. Serano R.c
}

Fecha de Recepción: 17 - ene. - 2018.

Fecha de Aceptación: 10 - may. - 2018.

\begin{abstract}
Resumen
El desaprovechamiento del recurso eólico en los países emergentes no es solo por la falta de la implementación de parques de aerogeneradores de gran escala, sino porque, en la mayoría de los territorios las velocidades promedio del viento no alcanzan a contar con la magnitud mínima ( 3 a $4 \mathrm{~m} / \mathrm{s}$ ) de arranque de los aerogeneradores comerciales, lo cual hace que este recurso no sea aprovechado. Por lo anterior, en el presente artículo se describe el diseño y caracterización de un elemento mecánico (concentrador eólico) que permite aumentar la potencia eólica disponible en un aerogenerador de baja potencia. El estudio está fraccionado en tres etapas: en la primera etapa se realiza un análisis experimental con el fin de validar e identificar el perfil de velocidades circundantes en el concentrador. En la segunda etapa se realiza un modelado mediante la ecuación de 5to. orden de Bell \& Mehta, implementando Matlab y exportando los datos a Solid Edge. Además, se describe el fenómeno mediante la ecuación de conservación de masa y conservación de energía. En la tercera etapa se realiza la simulación mediante elementos finitos soportado por el software ANSYS, la cual tiene como objetivo determinar la relación de área óptima de entrada y salida del concentrador. Finalmente, se concluye que el concentrador tiene un aporte significativo en el aumento del potencial eólico disponible del orden 2.37 veces.
\end{abstract}

Palabras clave: concentrador, eólico, simulación.

a Ingeniero Mecánico $@$, Universidad de Pamplona. esneider.arciniegas@unipamplona.edu.co

b Profesor Titular, Universidad de Pamplona. eflorez@unipamplona.edu.co

c Profesor Asociado, Universidad de Pamplona. jcserrano@unipamplona.edu.co 


\begin{abstract}
The waste of the wind resource in emerging countries is not only due to the lack of implementation of large-scale wind farms of, but because, in the most of territories the average wind speeds do not reach to the minimum magnitude ( 3 at $4 \mathrm{~m} / \mathrm{s}$ ) of starting of the commercial wind turbines, which means that this resource is not used. Therefore, in this article we describe the design and characterization of a mechanical element (wind concentrator) that allows increasing the wind power available in a low power wind turbine. The study is broken down into three stages: in the first stage an experimental analysis is conducted in order to validate and to identify the profile of surrounding velocities in the concentrator. In the second stage, a modeling is carried out throw the 5th order equation of Bell \& Mehta, by implementing Matlab and exporting the data to Solid Edge. In addition, the phenomenon is described by the equation of conservation of mass and conservation of energy. In the third stage the simulation is carried out by means of finite elements supported by the software ANSYS, whose objective is to determine the relation of the optimal area of entrance and exit of the concentrator. Finally, it is concluded that the concentrator allows the increasing of the wind potential of the order 2.37 times.
\end{abstract}

Keywords: concentrator, wind, simulation.

\section{INTRODUCCIÓN}

En el informe presentado por la ex primera ministra de noruega Gro Harlem Brundtland ante la ONU se nombra por primera vez el término desarrollo sostenible, el cual se define como: "la satisfacción de las necesidades de la generación presente sin comprometer la capacidad de las generaciones futuras para satisfacer sus propias necesidades" (ONU, 1987). De igual forma, según las Naciones Unidas, el cambio climático supone efectos adversos, referidos a cambios en el medio ambiente físico o en la biota con consecuencias nocivas significativas en la composición, la capacidad de recuperación o la productividad de los ecosistemas naturales o sujetos a ordenación, o en el funcionamiento de los sistemas socioeconómicos, o en la salud y el bienestar humano (Pinto y Salazar, 2014). Por consiguiente, uno de los objetivos mundiales en pro del desarrollo sostenible es "Garantizar el acceso a una energía asequible, fiable, sostenible y moderna para todos" (PNUD, 2015), por lo tanto, los países en vía de desarrollo tienen un mayor compromiso para lograr esta meta. Paralelamente, el Congreso Colombiano decretó mediante la ley 1715 del 2014 "promover el desarrollo y la utilización de las fuentes no convencionales de energía, principalmente aquellas de carácter renovable" (COLOMBIA, 2014). Por todo lo anterior, es importante desarrollar tecnología que mejore los sistemas de generación y transformación de energía renovable.

Colombia está en un punto intermedio entre los trópicos de cáncer y capricornio, lo que indica que es una zona de alta radiación solar e incidencia de vientos alisios del noreste, vientos constantes que recorren estas latitudes a una velocidad media de $5.55 \mathrm{~m} / \mathrm{s}$. Sin embargo, la orografía y otros factores modifican las corrientes atmosféricas cambiando la condición natural de los vientos a velocidades inferiores de la media (GÓMEZ, 2012).

En consecuencia, se estudia la forma de aumentar la potencia de un sistema eólico, utilizando para ello el Principio Venturi en los vientos que impulsan el aerogenerador. Asimismo, se utilizan las velocidades del viento presentes en el territorio Colombiano iguales e inferiores a $3.5 \mathrm{~m} / \mathrm{s}$ (Ver figura 1), siendo esta la velocidad mínima promedio a la que arrancan los aerogeneradores comerciales. 

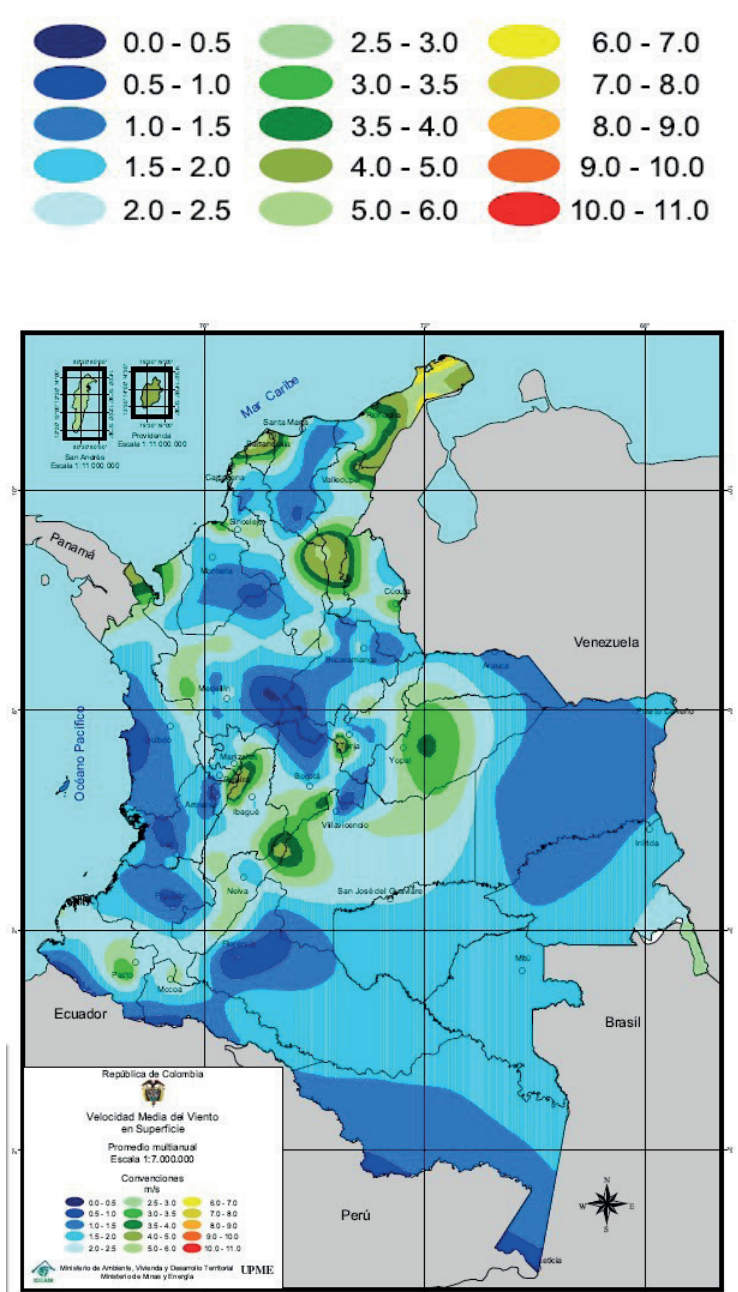

Figura 1. Atlas de viento y energía eólica de Colombia. Promedio multianual. (m/s). Fuente: UPME.

El concentrador de flujo eólico fundamenta su diseño en un túnel cónico con dos secciones circulares abiertas y transversales a la dirección del viento, en el cual se aplica el efecto Venturi; de tal forma, que el aerogenerador se ubica en la sección menor del túnel donde se ve aumentada la velocidad del viento, por el principio de continuidad. Al respecto concierne decir, que el potencial eólico por unidad de área es proporcional a la densidad y a la velocidad del viento al cubo, lo que indica, que si se aumenta la velocidad del viento en el paso por el aerogenerador, se incrementa en un orden cúbico el potencial eólico disponible.

\section{METODOLOGÍA}

Para realizar el estudio del concentrador eólico se asumieron tres etapas:

- $\quad$ Etapa 1. Análisis experimental.

- Etapa 2. Modelado.

- Etapa 3. Simulación.

\subsection{Análisis experimental}

El análisis se realiza bajo un modelo a escala del sistema eólico, teniendo presente el análisis de antecedentes e investigativo (Bell \& Mehta). En el experimento se evalúan las velocidades del viento tanto en la entrada como en la salida del concentrador con el objetivo de identificar el perfil de velocidades circundantes que se generan en el concentrador para una posterior validación. El ensayo está compuesto por los siguientes elementos:

- Un anemómetro CK120197.

- Un concentrador eólico con un ángulo de ataque de $20.5^{\circ}$ y con una relación de áreas de 4.

- Un ventilador de piso industrial de marca Home Elements HEHAF-18.

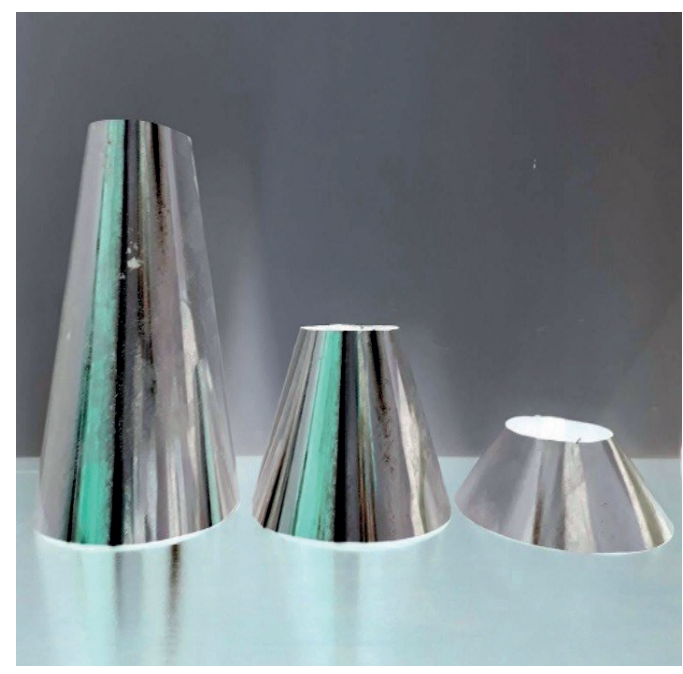

Figura 2. Modelo básico del concentrador. 
En las figuras 3 y 4 se ilustran los perfiles de velocidades obtenidos del experimento.

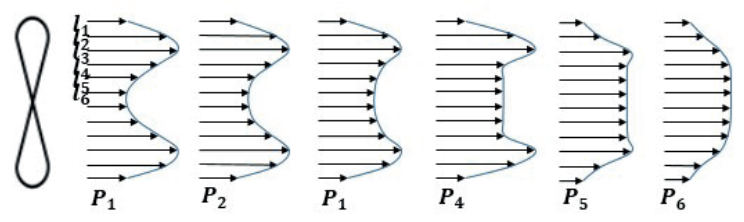

Figura 3. Perfil de velocidades circundantes.

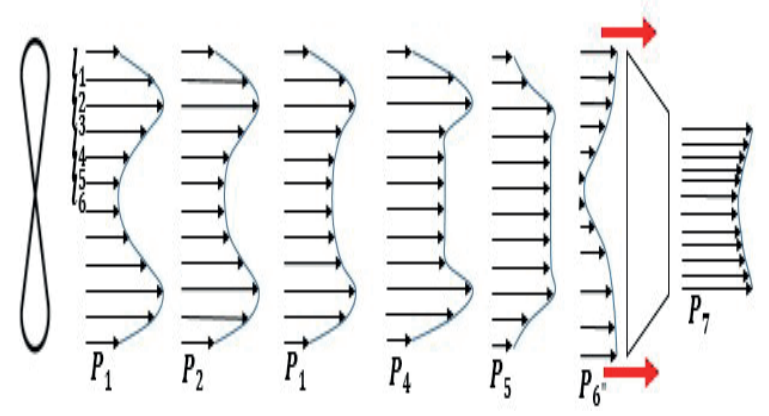

Figura 4. Perfil de velocidades circundantes con el concentrador.

De las figuras 3 y 4 se observa que el perfil P6 es alterado por la disposición del concentrador eólico, dando como resultado el perfil P6". Esto indica, que las velocidades en la entrada del concentrador son inferiores a las velocidades circundantes en el ambiente (Ver tabla 1). Sin embargo, las velocidades a la salida del concentrador son superiores en orden de $0.9 \mathrm{~m} / \mathrm{s}$ a las velocidades circundantes del ambiente.

Tabla 1. Nombre de la tabla.

\begin{tabular}{ccccccccc}
\hline Lc & P1 & P2 & P3 & P4 & P5 & P6 & P6" & P7 \\
\hline L1 & 2.1 & 1.8 & 1.5 & 1.4 & 1.2 & 1.1 & 2.2 & 4.0 \\
\hline L2 & 4.2 & 3.9 & 3.6 & 3.0 & 2.1 & 2.1 & 1.8 & 3.8 \\
\hline L3 & 4.5 & 4.4 & 4.2 & 4.0 & 3.5 & 3.1 & 1.4 & 3.7 \\
\hline L4 & 3.7 & 3.7 & 3.4 & 3.4 & 3.3 & 3.2 & 0.9 & 3.5 \\
\hline L5 & 2.5 & 2.8 & 3.0 & 3.3 & 3.3 & 3.2 & 0.4 & 3.4 \\
\hline L6 & 2.1 & 2.4 & 2.7 & 3.3 & 3.3 & 3.2 & 0.2 & 3.2 \\
\hline Pro. & 3.2 & 3.2 & 3.0 & 3.0 & 2.8 & 2.7 & 1.2 & 3.6 \\
\hline
\end{tabular}

\subsection{Modelado}

Para determinar la longitud de operación se siguen los parámetros de Bell \& Mehta. Donde:

$$
0.89 \leq \frac{L}{H_{e}} \leq 1.79
$$

Por lo tanto, para no estar al mínimo de generar números de Reynolds altos se promedian los límites de la longitud de operación (Longitud larga: genera el crecimiento de la capa límite. Longitud corta: genera disturbios en la uniformidad del flujo a medida que la sección transversal cambia (MOLINA, 2012)) adquiriendo una longitud óptima para la geometría del concentrador. Permitiendo obtener la tabla 2.

Tabla 2. Longitudes óptimas vs. relación de áreas.

\begin{tabular}{ccc}
\hline $\begin{array}{c}\text { Relación de } \\
\text { áreas }\end{array}$ & $\begin{array}{c}\text { Longitud } \\
\text { óptima (m) }\end{array}$ & $\begin{array}{c}\text { Relación de } \\
\text { longitudes }\end{array}$ \\
\hline$\frac{A_{1}}{A_{2}}=R_{A}$ & $L$ & $\frac{L}{\emptyset_{2}}=R_{L}$ \\
\hline 2 & 1.12 & 0.96 \\
\hline 3 & 1.35 & 1.15 \\
\hline 4 & 1.57 & 1.34 \\
\hline 5 & 1.74 & 1.48 \\
\hline 6 & 1.91 & 1.63 \\
\hline 7 & 2.06 & 1.76 \\
\hline $4 \mathrm{C}$ & 1.04 & 0.88 \\
\hline $4 \mathrm{~L}$ & 2.08 & 1.78 \\
\hline
\end{tabular}

Para obtener el perfil de contracción se acude a la ecuación polinomial de quinto orden de Bell $\&$ Mehta, la cual tiene como objetivo principal minimizar la longitud de contracción de manera que se obtenga un mínimo de Reynolds a la salida.

$$
\begin{gathered}
\begin{array}{c}
\text { Ecuación de 5to. orden de Bell \& Mehta. } \\
\text { (MEHTA, 1988) }
\end{array} \\
h=H_{e}-\left(H_{e}-H_{S}\right)\left(6 \xi^{5}-15 \xi^{4}+10 \xi^{3}\right) \\
\xi=\frac{x}{L}
\end{gathered}
$$

Para adquirir el perfil de la contracción se utiliza el software de Matlab, el cual permite procesar los datos. Una vez desarrollado el código en Matlab se obtienen las coordenadas y se exportan a Solid Edge (Ver figura 5 y 6, se ilustran los perfiles de contracción para cada relación de áreas). 


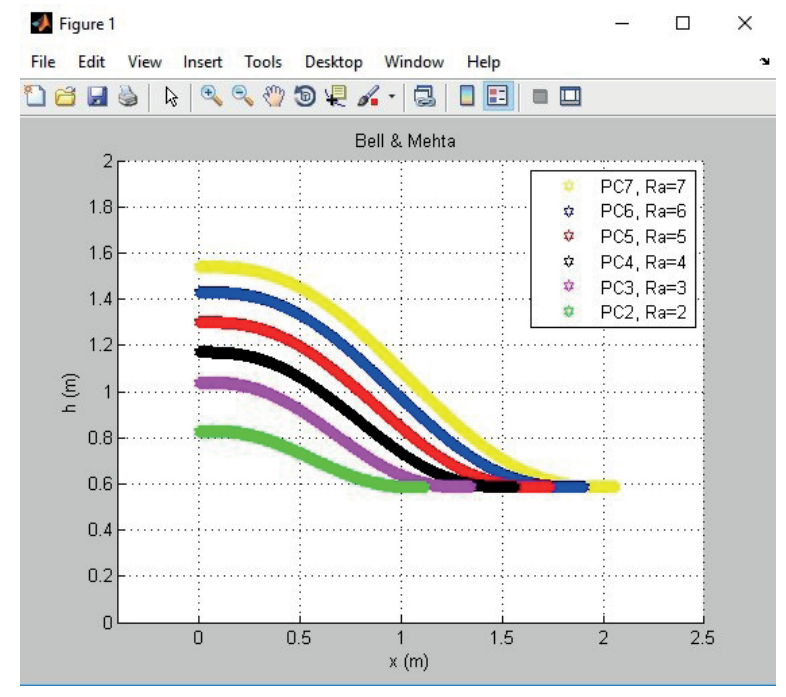

Figura 5. Perfiles de contracción de relaciones de área de 2 hasta 7.

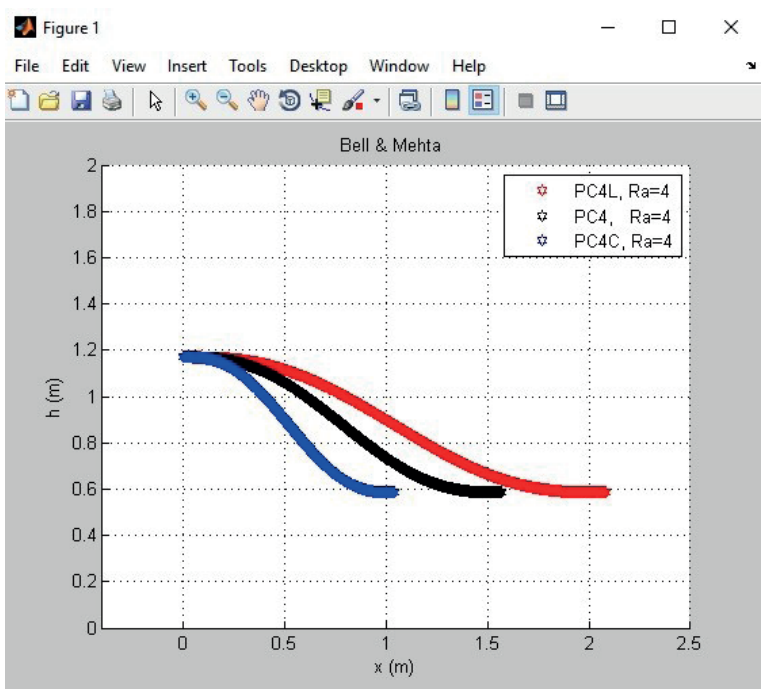

Figura 6. Perfiles de contracción de relación de área de 4 llevando al límite la longitud de operación.

Los datos exportados son 1000 coordenadas en los ejes X y Y. Se adicionan 1000 coordenadas de $Z$ cero para ubicar el perfil en el boceto de solid edge.

Una vez almacenados los datos se procede a realizar el modelo del concentrador de flujo eólico mediante las herramientas de Solid Edge (Ver figura 7).

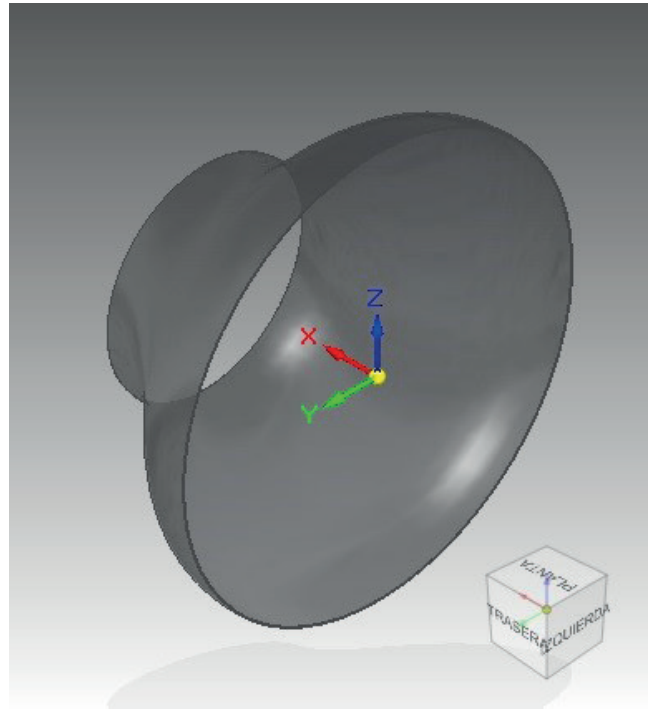

Figura 7. CAD del Perfil del Concentrador 4 Corto (PC4C).

El flujo másico es el mismo en todas las secciones del concentrador de flujo eólico. Por ende, se debe cumplir la ecuación de conservación de masa para el sistema. (Ver figura 8).

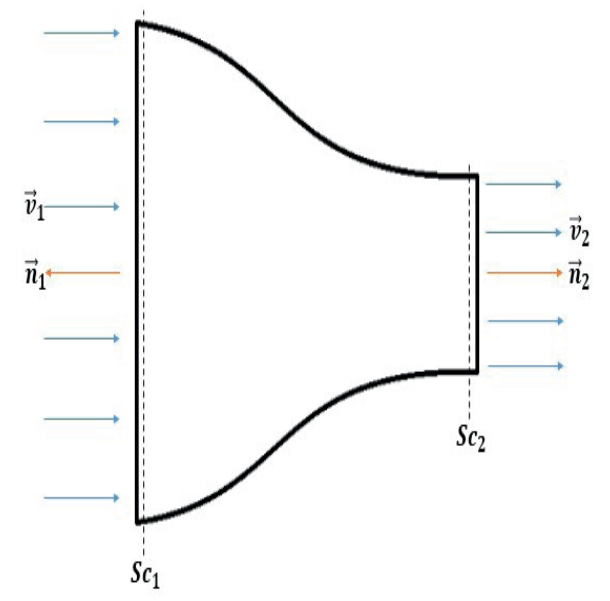

Figura 8. Sistema de control

Asumiendo que las propiedades del fluido permanecen constantes en el tiempo y partiendo de que el concentrador eólico tiene dos superficies de control; entonces, se puede despreciar la parte temporal y simplificar la ecuación a: 
Una vez conocidas las velocidades y las perdidas por presión según la tabla de Kisieliev, se realiza un balance de energía para determinar la presión de salida mediante la siguiente ecuación.

$$
\begin{gathered}
\int_{\gamma C} \frac{\partial \rho}{\partial t} d V+\int_{S C} \rho \vec{v} \cdot \vec{n} d A=0 \\
\int_{S c_{1}} \rho \vec{v} \cdot \vec{n} d A+\int_{S c_{2}} \rho \vec{v} \cdot \vec{n} d A=0
\end{gathered}
$$

\subsection{SIMULACIÓN}

El análisis de fluidos en el concentrador de flujo eólico se realiza mediante simulaciones en CFD, ANSYS Workbench.

El dominio computacional tiene una forma cilíndrica con medidas de 6 veces la longitud de la geometría aguas abajo, 3 veces aguas arriba y con un diámetro de 5 veces de la sección trasversal del sólido (Ver figura 9).

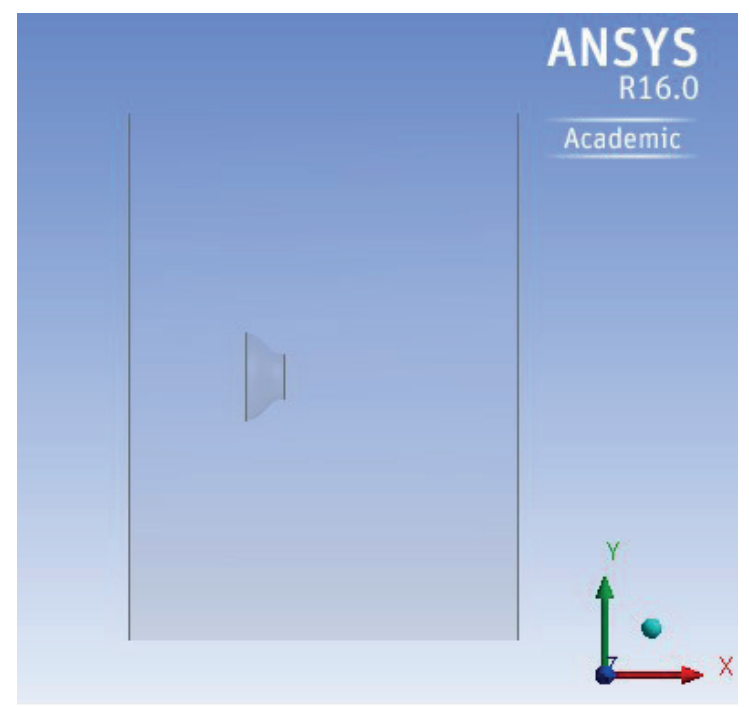

Figura 9. Dominio computacional.

El mallado híbrido está conformado por elementos tetraédricos para el volumen del fluido en general y prismáticos para la captura del comportamiento de la capa límite adyacente a la pared del concentrador. Los elementos de la malla global están conformados por: tamaño mínimo de $4 \mathrm{~mm}$, tamaño máximo de $120 \mathrm{~mm}$, tasa de crecimiento para las celdas en el dominio de 1.2 y una transición suave con 5 capas de inflación con una tasa de crecimiento 0.172 (Ver figura10). No se presentó la necesidad de realizar mallas locales.

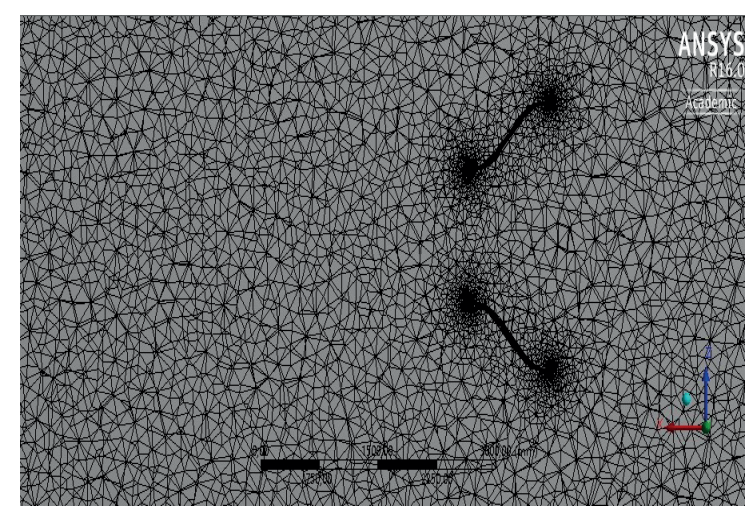

Figura 10. Mallado del PC4C.

La cantidad de los nodos y elementos están sujetos a la disposición de la geometría y el dominio computacional. De los cuales, 3348420 son tetraédricos y 662920 prismáticos, conectados por 952537 nodos.

La calidad ortogonal alcanzó un rango muy bueno con un promedio de 0.86 . Donde, 0 es inaceptable y 1 es excelente. De igual forma, la oblicuidad obtuvo un rango excelente con un promedio de 0.22 . Donde, 1 es inaceptable y 0 es excelente.

Análogamente, se establece el Shear Stress Transporte SST $K-\omega$ como modelo de turbulencia con un fluido de densidad $1.2 \mathrm{~kg} / \mathrm{m}^{3}$ y viscosidad dinámica de $1.8 \times 10^{-5} \mathrm{~N} \mathrm{~s} / \mathrm{m}^{2}$. Simultáneamente, la velocidad de entrada se establece constante con un valor de $3 \mathrm{~m} / \mathrm{s}$ y es perpendicular a la superficie de la entrada del dominio computacional con una intensidad de turbulencia porcentual de 2.6. También, se establece una presión a la salida de $0 p a$.

Se selecciona el algoritmo de solución SIMPLE-C para el acoplamiento presión - velocidad, el cual se programa para 1000 iteraciones, en el cual la solución presenta convergencia a las 516 iteraciones con un criterio de convergencia de 1e- 6 para velocidades en $\mathrm{X}$.

Como resultado de la simulación se muestran seis líneas internas (series) de las velocidades del fluido en el concentrador (Ver figura 11), con una velocidad inicial de $3 \mathrm{~m} / \mathrm{s}$. las velocidades 
en la entrada $(0 \mathrm{~m})$ del concentrador están en el orden de $1.7 \mathrm{~m} / \mathrm{s}$ y a la salida $(1.04 \mathrm{~m})$ del mismo alcanzan valores de $3.9 \mathrm{~m} / \mathrm{s}$. Sin embargo, 20 cm después de la salida del concentrador, estas velocidades están por el orden de $4 \mathrm{~m} / \mathrm{s}$. Las velocidades a la salida presentan una tolerancia aproximada de $0.05 \mathrm{~m} / \mathrm{s}$, lo que indica, que estas velocidades muestran una homogeneidad en toda el área de salida.

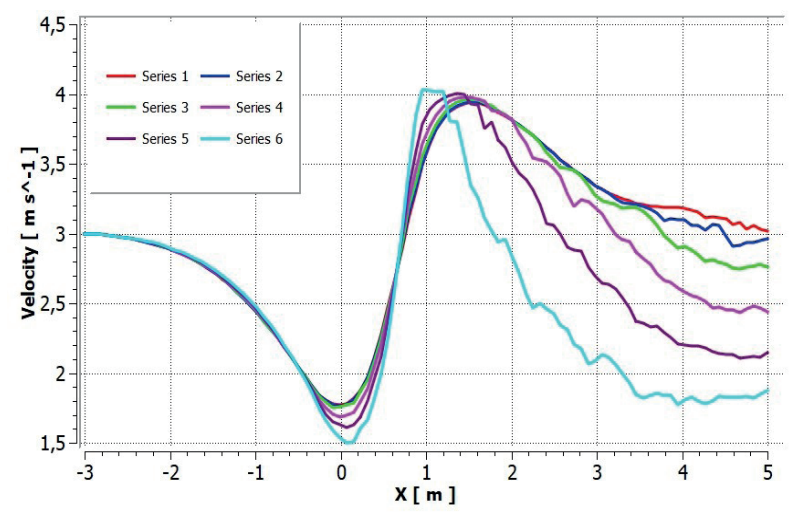

Figura 11. Series de velocidades del fluido.

En la figura 12 se ilustran las posibles trayectorias que pueden seguir las partículas del fluido para una velocidad inicial de $3 \mathrm{~m} / \mathrm{s}$, estas trayectorias asignan magnitudes superiores a $4 \mathrm{~m} / \mathrm{s}$. El flujo se establece como estado estacionario.

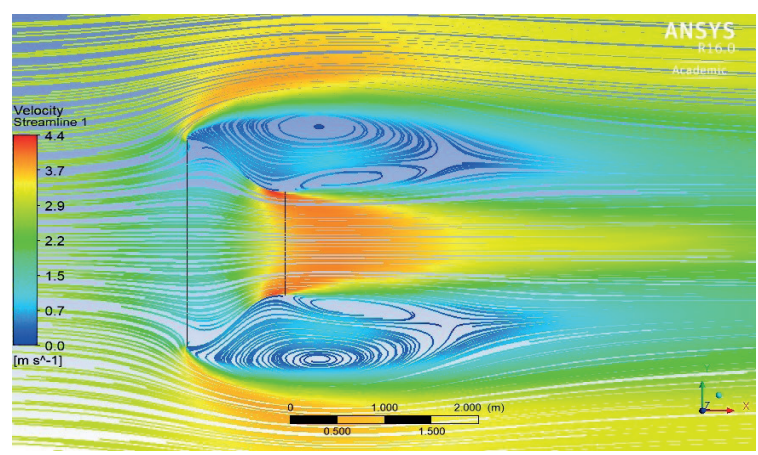

Figura 12. Líneas de corriente.

Los mapas de contorno (Ver figura 13 y 14) ilustran que las velocidades en la entrada del concentrador son inferiores a las velocidades circundantes del ambiente $(3 \mathrm{~m} / \mathrm{s})$. Lo que indica, que el concentrador en la entrada actúa como una resistencia al paso del fluido. Sin embargo, las velocidades máximas están por el orden de $4 \mathrm{~m} / \mathrm{s}$ cerca a la salida del concentrador, debido al efecto Venturi. Estas velocidades son homogéneas a cierta distancia en la cual se podría ubicar el rotor del aerogenerador.

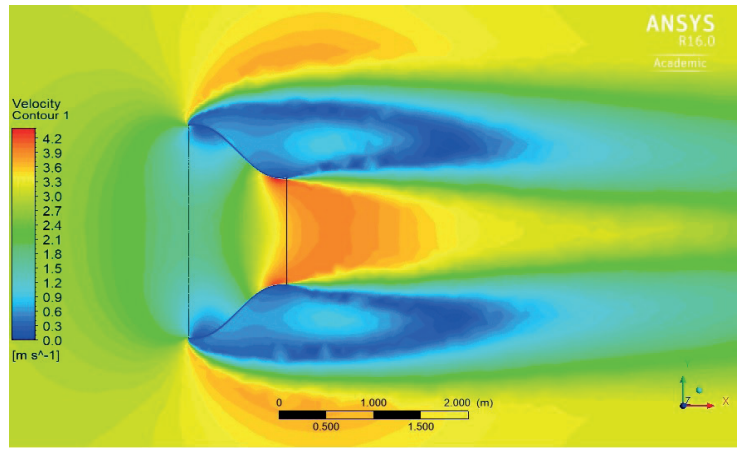

Figura 13. Mapa de contorno / Velocidades del fluido, vista lateral del PC4C.

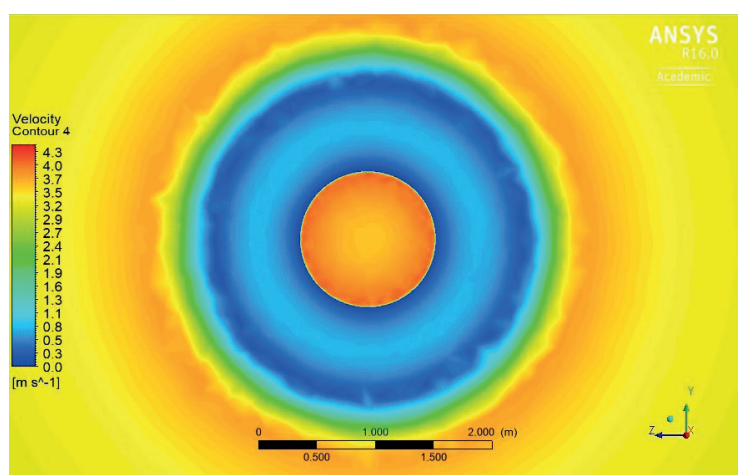

Figura 14. Mapa de contorno / velocidades a la salida del PC4C.

Las figuras 15 y 16 ilustran las presiones presentes en las líneas internas del concentrador, estas presiones toman valores positivos antes de $0.8 \mathrm{~m}$ en el interior del ducto, debido a que las velocidades del fluido han disminuido en la entrada. Después de esta distancia toman valores negativos dado que las velocidades han aumentado por encima de $\operatorname{los} 3 \mathrm{~m} / \mathrm{s}$. Lo que indica, que a medida que incrementa la velocidad disminuye la presión.

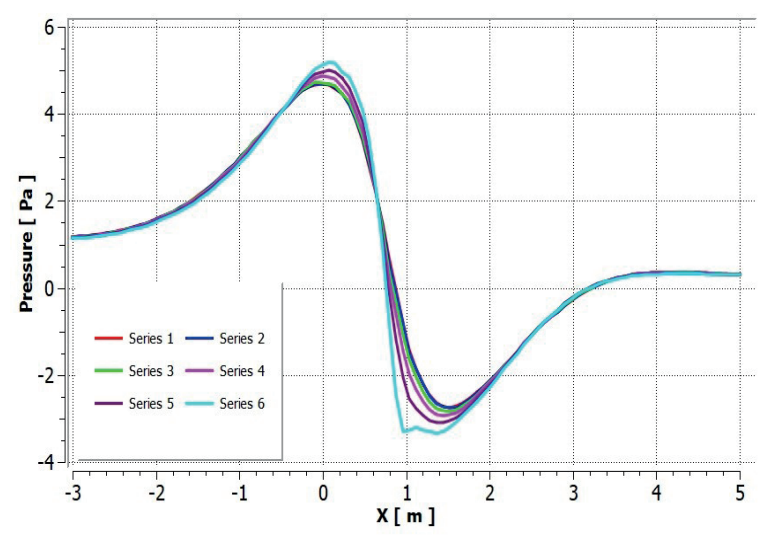

Figura 15. Series de presión del fluido. 


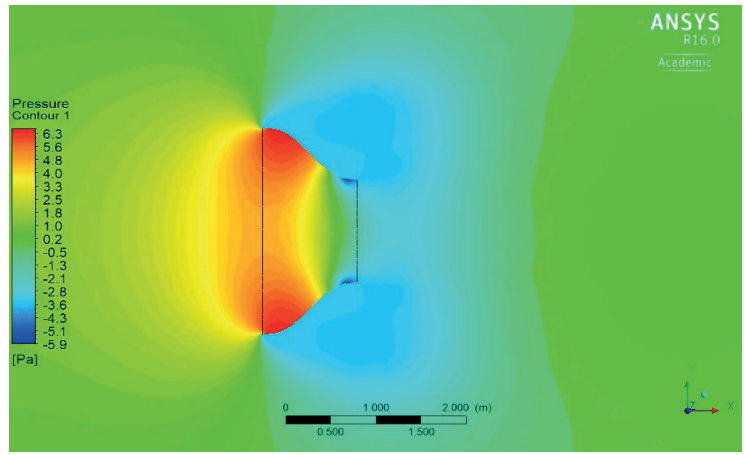

Figura 16. Mapa de contorno / Presión del fluido.

\section{RESULTADOS}

Para la validación de los resultados se toman los datos obtenidos en el desarrollo experimental y los obtenidos en la simulación de prueba. En el cual, el promedio de las velocidades circundantes del ambiente de la parte experimental está en 2.7 $\mathrm{m} / \mathrm{s}$ y el promedio de velocidades a la salida del concentrador "modelo" está en un valor de 3.6 $\mathrm{m} / \mathrm{s}$, el cual se coteja con el promedio de velocidades a la salidad de la simulación de prueba de 3.4 $\mathrm{m} / \mathrm{s}$. determinando un error relativo porcentual de 5.5 .

Una vez realizada la simulación de prueba y la validación de los datos, se efectúan las simulaciones para el diseño del concentrador. Donde, inicialmente se realizan 6 simulaciones con diferentes relaciones de áreas.

Análogamente, se comparan los resultados de las seis simulaciones entre sí determinado que el Perfil del Concentrador 4 (PC4) presenta velocidades considerables a la salida con menores dimensiones de diseño (volumen y material). Sin embargo el PC5, PC6 y PC7 presenta un ligero aumento a la salida, pero no justifica tal aumento respecto al volumen del concentrador (Ver figura 17).

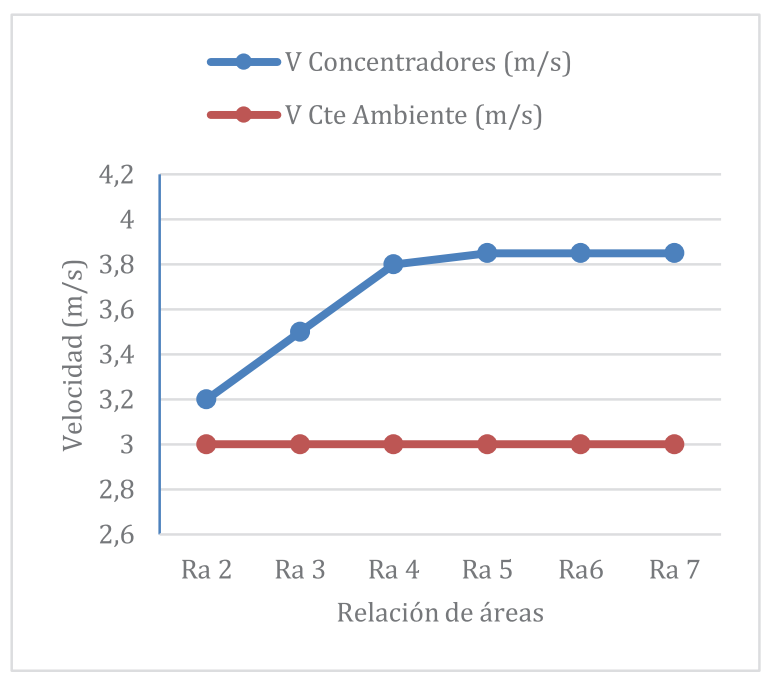

Figura 17. Relación de diseño de 2 hasta 7 vs. velocidad.

Posteriormente, se realizan dos simulaciones más al PC4, con la diferencia que se lleva al límite la longitud de operación del concentrador con base en los parámetros de Bell \& Mehta. En el cual, se logra determinar que el Perfil del Concentrador 4 Corto (PC4C) genera mayores velocidades a la salida del orden de $4 \mathrm{~m} / \mathrm{s}$, optimizando así la longitud del mismo (Ver figura 18).

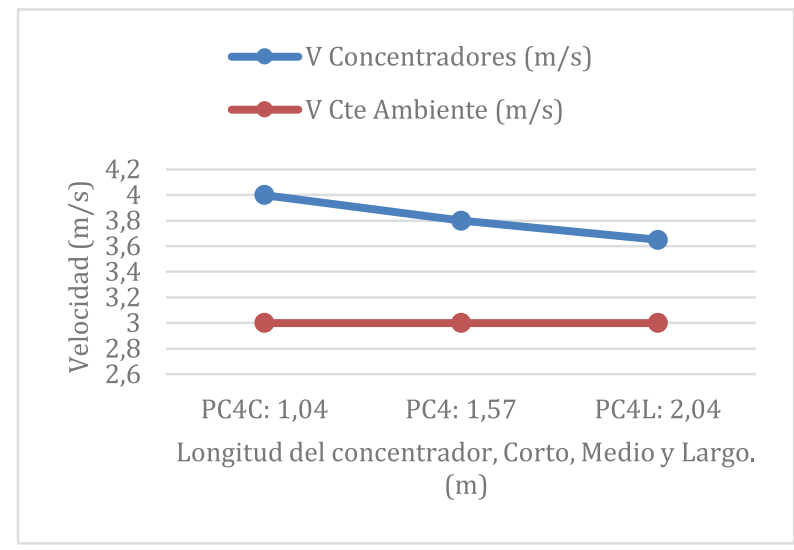

Figura 18. Relación de velocidad vs. diseño del PC4 llevado hasta el límite de operación. 


\section{DISCUSIÓN}

La validación de las simulaciones en los diseños finales se asume sobre el comportamiento semejante en la solución del algoritmo de acoplamiento presión-velocidad a la simulación de prueba. No obstante, se debe tener presente que esta última se validó con el análisis experimental, donde dicho análisis se valora de manera empírica debido a las limitaciones de los recursos.

\section{CONCLUSIONES}

Se realizaron un total de 9 simulaciones, la primera de ellas está orientada a la validación del análisis experimental, las siguientes 6 están enfocadas a identificar la relación de área que aporte mayores velocidades a la salida del concentrador eólico con los mínimos recursos de diseño y las 2 últimas están encaminadas a calcular el aumento de la velocidad respecto a la variación de la longitud de operación de Bell \& Mehta. Por último, se determina que el concentrador $\mathrm{PC} 4 \mathrm{C}$ presenta un aumento de $1 \mathrm{~m} / \mathrm{s}$ las velocidades del fluido (Aire), llevando la potencia eólica disponible de a, lo que representa un incremento 2.37 veces.

El concentrador eólico PC4C tiene como dimensiones, una longitud axial de $1.04 \mathrm{~m}$ con una relación de áreas de 4 , siendo su diámetro de salida de $1.17 \mathrm{~m}$. Lo que permite menores volúmenes de diseño respecto al PC4, PC4L, PC5, PC6 y PC7.

La mayoría de los aerogeneradores comerciales de baja potencia requieren una velocidad mínima de arranque de $3.5 \mathrm{~m} / \mathrm{s}$. No obstante, las velocidades del viento en el territorio de Colombia están por el orden de los $3 \mathrm{~m} / \mathrm{s}$ según el mapa de vientos de la UPME. Esto implicaría que se requieren mayores velocidades para poner en marcha a los aerogeneradores. Sin embargo, el concentrador eólico permitiría poner en funcionamiento a los aerogeneradores con estas velocidades, debido a que este aumenta los vientos a $4 \mathrm{~m} / \mathrm{s}$. Por lo tanto, se generarían mayores tiempos en el funcionamiento.

\section{BIBLIOGRAFÍA}

[1] COLOMBIA, C. D. (2014). LEY 1715 DE 2014 Diario Oficial No. 49.150. Bogotá, D.C.: Imprenta Nacional. Retrieved 09 2017, from http://www.comunidadcontable.com/ BancoMedios/Imagenes/ley\%201715\%20 de\%202014.pdf

[2] GÓMEZ, C. L. (2012). Clima ecuatorial. Climatología. Retrieved 10 2017, from http://titulaciongeografia-sevilla.es/ contenidos/profesores/materiales/archivos/2012-04-16CLIMAeCUATORIAL.pdf

[3] MEHTA, B. \&. (1988). Contraction desing for small low speed wind tunnels. In J. H. MEHTA, ACOUSTICS, JOINT INSTITUTE FOR AERONAUTICS (p. 14). Retrieved 09 04, 2017, from https://ntrs.nasa.gov/archive/ nasa/casi.ntrs.nasa.gov/19880012661.pdf

[4] MOLINA, F. E. (2012). Diseño y construcción de un túnel de viento (Universidad Centroamérica "José Simeón Cañas" ed.). Salvador. Retrieved 2017, from http://cef. uca.edu.sv/descargables/tesis_descargables/ tunel.pdf

[5] ONU, G. H. (1987). El informe Brundtland. Retrieved 09 2017, from http://www.un.org/ es/ga/president/65/issues/sustdev.shtml

[6] PINTO H. J. E., SALAZAR DE CARDONA M. (2014). Cambio climático y vulnerabilidad: prospectivas para la región nororiental de Colombia - Santanderes, Revista BISTUA. Vol. 12 (1).

[7] PNUD, P. D. (2015). Objetivos de Desarrollo Sostenible, Colombia. Herramientas de aproximación al contexto local. Retrieved 09 2017, from http://www.co.undp.org/content/colombia/es/home/post-2015/sdg-overview/goal-7.html 
